\title{
Superconducting Storage Cavity for RHIC
}

\author{
I. Ben-Zvi
}

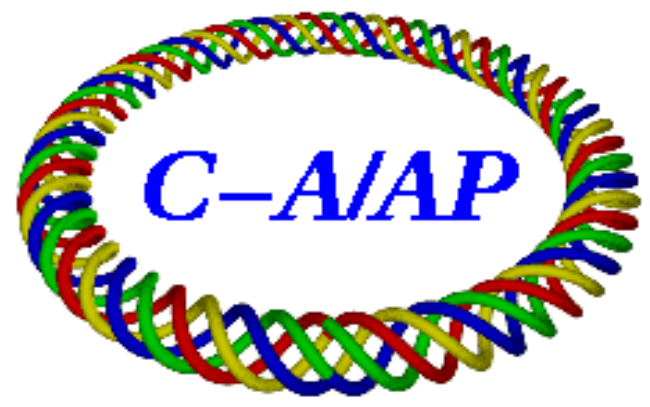

\section{Collider-Accelerator Department Brookhaven National Laboratory Upton, NY 11973}

Notice: This document has been authorized by employees of Brookhaven Science Associates, LLC under Contract No. DE-AC02-98CH10886 with the U.S. Department of Energy. The United States Government retains a nonexclusive, paid-up, irrevocable, world-wide license to publish or reproduce the published form of this document, or allow others to do so, for United States Government purposes. 


\title{
Superconducting Storage Cavity for RHIC
}

\author{
Ilan Ben-Zvi, Collider-Accelerator Department, Brookhaven National Laboratory
}

\begin{abstract}
This document provides a top-level description of a superconducting cavity designed to store hadron beams in the Relativistic Heavy Ion Collider (RHIC) at Brookhaven National Laboratory. It refers to more detailed documents covering the various issues in designing, constructing and operating this cavity.

The superconducting storage cavity is designed to operate at a harmonic of the bunch frequency of RHIC at a relatively low frequency of $56 \mathrm{MHz}$. The current storage cavities of RHIC operate at $197 \mathrm{MHz}$ and are normal-conducting. The use of a superconducting cavity allows for a high gap voltage, over $2 \mathrm{MV}$. The combination of a high voltage and low frequency provides various advantages stemming from the resulting large longitudinal acceptance bucket.
\end{abstract}

\section{Project Description}

A $56 \mathrm{MHz}$ superconducting cavity is being designed and built for the RHIC collider. The cavity will operate at harmonic 720 of the revolution frequency of RHIC, thus a more precise frequency is 56.29884 $\mathrm{MHz}$, depending weakly on the beam energy. The cavity will be placed in the common section of RHIC (where both blue and yellow ring have a common axis), so that it will provide a storage RF potential for both rings.

The low frequency of the cavity calls for a loaded design, in order to reduce the physical size of the cavity. This approach is common in superconducting low-energy heavy-ion linear accelerators. The shape chosen for the cavity is a quarter-wave resonator. This type of superconducting resonator is very common in heavy ion linacs, and it is also the design of the (normal-conducting) $28 \mathrm{MHz}$ accelerating cavity of RHIC. As in the RHIC accelerating cavity, but in contrast to heavy-ion linac cavities, the beam path is along the symmetry axis of the resonator, as can be seen in Figure 1 . The cavity is about 1.5 meters long along the beam axis and it is about $50 \mathrm{~cm}$ in diameter.

The cavity will be made of niobium, and it is expected that a voltage of about $2 \mathrm{MV}$ to $2.5 \mathrm{MV}$ will be maintained across its single gap.

The low frequency (RHIC harmonic $h=720$ ) and large voltage $(\mathrm{V}=2.5 \mathrm{MV}$ ) of the cavity will provide for a large longitudinal bucket $A_{0}$, which will reduce spill from the bucket due to Intra-Beam Scattering (IBS) and thus reduce the background in the detectors.

The size of the bucket using the $56 \mathrm{MHz}$ cavity at RHIC is $6.4 \mathrm{eV}^{*} \mathrm{~s} / \mathrm{u}$ for $100 \mathrm{GeV} / \mathrm{u}$ gold ions and 15.5 $\mathrm{eV}^{*} \mathrm{~s}$ for $250 \mathrm{GeV}$ protons, in both cases much larger than the beam emittance. 


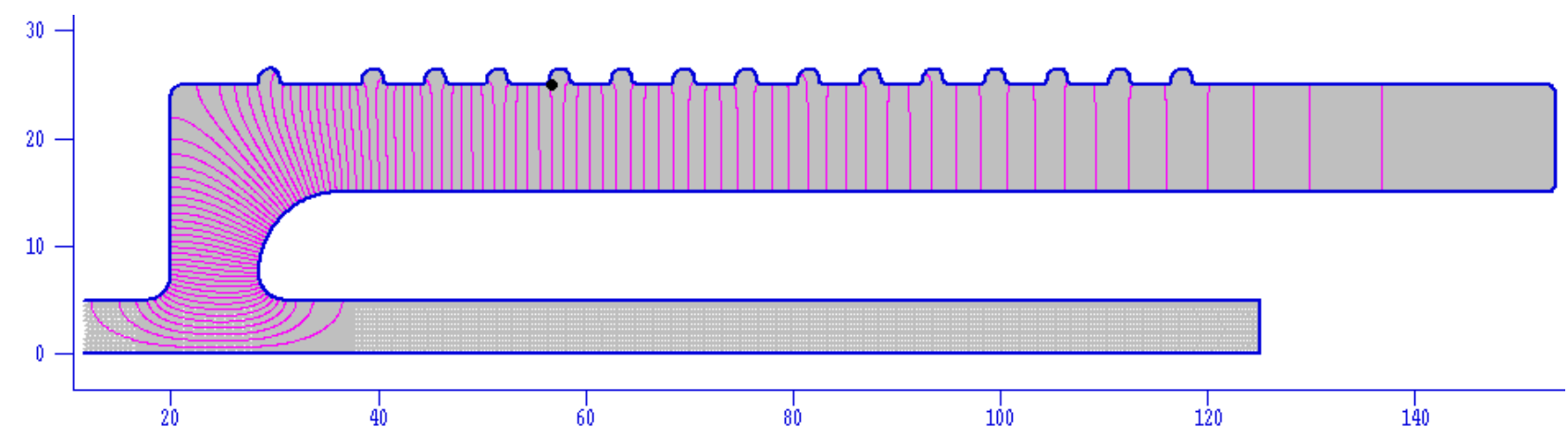

Figure 1. The $56 \mathrm{MHz}$ cavity, as drawn by the program SUPERFISH [Ref Chang] and it shows electric field lines in the cavity. The beam axis is along the zero line of the vertical axis and it is a figure of revolution along the beam axis. The figure shows the optimized shape of the gap and corrugations along the outer conductor [Ref Naik] as will be discussed in the multipacting section.

This large bucket also allows for adiabatic rebucketing from the $28 \mathrm{MHz}$ cavity. This approach keeps all the ions in one bucket, thus reduce the effective bunch length, and thus increase luminosity in the vertex of the detectors.

The performance of RHIC with and without the $56 \mathrm{MHz}$ storage cavity has been analyzed in detail under various conditions. [Ref Fedotov]. The $56 \mathrm{MHz}$ cavity can lead to a large increase in luminosity, $50 \%$ or more, depending on the configuration of RHIC at the time of its implementation.

Additional advantages include the fact that SRF cavities enjoy best vacuum, therefore reducing the potential for the pressure rise in Sector 4, and providing somewhat lower RHIC impedance (fewer storage cavities).

The cavity is intended to be beam driven, although a low-power ( $1 \mathrm{~kW}$ ) RF amplifier will be provided to provide amplitude control. The cavity-ring stability issues are discussed below.

The resonator will be operated at near atmospheric helium pressure, or at about 4.2 degrees $\mathrm{K}$ or somewhat above. This is possible due to its very low frequency, which makes the BCS surface resistivity negligibly small compared to the expected residual surface resistance, which is expected to be in the range of a few $n \Omega$, most likely below $10 \mathrm{n} \Omega$.

\section{The choice of the Resonator Shape and its Properties}

As mentioned above, the $56 \mathrm{MHz}$ cavity will be a quarter-wave resonator, with the beam traversing the symmetry axis of the resonator. The quarter-wave resonator is a very compact, yet efficient (high shunt impedance) shape. For a velocity-of-light particles, the axial orientation, with its single gap, provides a good transit-time factor.

Using the SUPERFISH code, we get [Ref Chang] the electrical parameters of the cavity for a gap voltage of $2.5 \mathrm{MV}$. In the power and $\mathrm{Q}$ calculation we assume a residual resistivity of $10 \mathrm{n} \Omega$. Additional parameters, related to electro-mechanical aspects, were calculated using the ANSYS code [Ref Pai]. 
Table 1. Electromagnetic and electromechanical parameters of the $56 \mathrm{MHz}$ SRF cavity

Stored energy

Operating temperature

Power dissipation

$\mathrm{Q}_{0}$

Design loaded Q

r/Q (acceleration notation)

Maximum surface magnetic field

Maximum surface electric field

Tuning rate of mechanical tuner

Elastic tuning range

Plastic tuning range

Lorentz detuning at 2.5 MV

First mechanical mode frequency

Piezoelectric tuner range

Piezoelectric tuner natural resonance $160 \mathrm{~Hz}$

$4.2 \mathrm{~K}$

$410^{7}$

$81 \Omega$

$95 \mathrm{kHz}$

$231 \mathrm{~Hz}$

$25 \mathrm{~Hz}$
Pressure frequency detuning

216 Joules (At 2.5 MV gap voltage)

$42 \mathrm{~W}$ (Assuming $10 \mathrm{n} \Omega$ residual surface resistivity)

$1.810^{+9}$ (Same assumption as above)

$84 \mathrm{kA} / \mathrm{m}$

$44 \mathrm{MV} / \mathrm{m}$

$15.8 \mathrm{kHz} / \mathrm{mm}, 0.016 \mathrm{~Hz} /$ step, $790 \mathrm{~Hz} /$ second

$>24 \mathrm{kHz}$

$0.28 \mathrm{~Hz} / \mathrm{mB}$

$98.5 \mathrm{~Hz}$

It is worth noticing that the resonator is beam driven, and has a large Lorentz frequency shift and a rather small loaded bandwidth. A monotonic instability is not an issue since the resonant frequency is approaching the generator (beam) frequency from below, however amplitude feedback is necessary to provide stability against the oscillatory ponderomotive instability.

The work referenced above provides details of the optimization method and also provides the details of setting the room temperature on-the-bench frequency right to obtain the desired cold, operational frequency.

- The shape of the resonator is affected by a few other considerations, some of which will be detailed in a few of the following sections. These include:

- The accessibility of the internal surface area to chemical polishing and high-pressure water rinsing, two important fabrication and preparation steps. 
- The compliance of the resonator and its helium vessel enclosure with the pressure vessel code.

- Tuning of the resonator has to be provided over sufficient range, yet be compliant with the pressure vessel code. The tuning range must be large enough to minimize the peak power absorbed by the fundamental mode damper while it is inserted as well as during its extraction.

- The high-electric field regions must be shaped to minimize the peak-surface-electric-field at a given voltage.

- The radial dimensions must avoid too high a peak-surface-magnetic-field.

- The resonator has to be designed to avoid multipacting. This leads to the corrugations that will be introduced below, but also to avoiding a tapered inner conductor.

- The resonator mechanical design must minimize the effect of helium pressure fluctuations on the resonator's frequency.

- The cryostat and cryogenic system must provide sufficient isolation of the resonator from environmental acoustic noise.

- The shape of the resonator must allow good cooling by pool-boiling liquid helium.

\section{Chemistry Issues}

The structure of the resonator has to accommodate filling the inner volume quickly with BCP acid mix and rapid removal of the acid. The acid must occupy the complete internal volume, leaving no trapped air. Then the resonator must accommodate the insertion of nozzles for high-pressure deionized water rinsing of the complete internal surface area. These constraints were realized by providing eight ports on the shorted-conductor end (furthest away from the accelerating gap) end of the cavity. These ports are also used for insertion of the Higher Order Mode (HOM) dampers, the fundamental power coupler and the pickup probe.

In addition, the shape of the fundamental damper port was chosen so as to be compatible with the chemistry process.

\section{Fundamental and HOM Dampers}

The design of the fundamental mode damper and the HOM dampers is the most challenging aspect of the cavity's design. The extensive effort is documented in a series of reports [Ref Choi]. The design provides sufficient damping of both fundamental mode and higher order modes, satisfying the stability criteria set [Ref Towne, Ref Blaskiewicz].

The injection energy of RHIC for gold ions is about $9.5 \mathrm{GeV} / \mathrm{u}$, or $\gamma=10.2$. Taking the circumference of RHIC as 3834 meters, the beam revolution frequency at injection is $7.782 * 10^{4} \mathrm{~Hz}$. At store, $\gamma^{\sim 100}$, the revolution frequency is $7.819 * 10^{4} \mathrm{~Hz}$. This difference is almost $5 \%$, or about $270 \mathrm{kHz}$, much more than 
the tuning range of the superconducting resonator. Therefore, as the beam will be accelerated from injection to store energy, the relative frequency of the beam to the cavity will be changing. Over this range, a few harmonics of the bunch revolution frequency will cross the cavity's frequency. Over part of this range, which is above the transition energy, the beam frequency will be on the wrong side of the cavity for Robinson stability considerations (more below). Please note that protons are injected above transition, so some of the considerations are slightly different. In any case, the fundamental mode of the cavity must be heavily damped while the beam is being accelerated in the RHIC rings. The loaded Q of the damped fundamental mode can be calculated from beam stability criteria, but a simpler constraint exists. The damped fundamental mode should not consume more than about $20 \mathrm{~kW}$ from the beam, since this power has to be put back into the beam by the accelerating cavity. In addition, we want to limit the power on the damping resistor. This calculation should be made at the maximum possible current of RHIC, and since the cavity will be seeing the current of both rings at the same time (being located at one of the common sections of RHIC), this is a rather significant constraint. The current driving the resonator field is $I_{b}=2 q f_{b}$ where $q$ is the bunch charge and $f_{b}$ is the bunch frequency. For protons with $2 \times 10^{11}$ particles per bunch, the bunch charge is $32 \mathrm{nC}$. For 112 bunches in one ring, or 224 in both rings, and a revolution frequency of $78 \mathrm{kHz}$, the current is about 1.1 amperes. Since the shunt impedance per $Q$ of the resonator is about $R / Q=40 \Omega$ (circuit definition), we can equate the maximum power of $P=20 \mathrm{~kW}$ to $P=0.5 \mathrm{~b}_{b}{ }^{2} R / Q^{*} Q=24 Q$, leading to a required $Q$ of less than 800 .

The fundamental mode damper dissipates a large power into its termination; however the current is modest (less than 20 amperes) for the large loop, which is $4 \mathrm{~cm}$ wide. In fact, most of the time the current is under 10 amperes. Thus the Joule heating of the coupler loop is negligible even when constructed out of copper. Similarly, at times that the damper is inserted into the cavity, the cavity is also detuned, resulting a negligible eddy current heating of the copper. If the copper maintains an emissivity of 0.1 , (polished copper has a significantly lower emissivity) then the loop can be maintained at $300 \mathrm{~K}$ with only about $1.2 \mathrm{Watt}$ radiated into the cavity. Thus conduction cooling of the fundamental mode damper is a reasonable approach.

The sequence of operation of RHIC with the $56 \mathrm{MHz}$ cavity will be as follows: When the beam is injected into RHIC, the fundamental mode damper will be fully inserted and the cavity will be tuned to its lower frequency limit. When the beam is completely injected and accelerated to the store energy, the fundamental mode damper will be moved out of the resonator while keeping the resonance frequency at its low end. Then the resonant frequency will be moved to the operational frequency, just below the resonance frequency and detuned just right to build the required voltage using the beam as a driver. The power amplifier will be used just to close an amplitude control feedback loop.

The Higher Order Mode (HOM) dampers will use a high-pass filter to avoid damping the fundamental mode but provide good damping of all relevant HOMs. One nice property of the quarter wave resonator is the large separation of the first HOM from the fundamental, allowing the use of a high-pass filter instead of the notch filter required in a TM010 type cavity.

The HOM dampers are inserted into the cavity while it is at full field. Thus the construction of the HOM dampers is from niobium. The fundamental current flowing in the high-pass filter is modest for a 
superconducting circuit, (up to 40 amperes), and the dissipation is low enough to allow us to maintain the HOM damper and its high-pass filter circuit at $4.2^{\circ} \mathrm{K}$ with conduction cooling. The HOM power will be deposited in an external load.

\section{Multipacting Issues}

While numerous niobium quarter-wave resonators were built and operated with reasonable conditioning of multipacting, a storage-ring accelerating cavity has to be ultimately reliable at all times. Therefore a new approach was taken toward eliminating multipacting in the resonator. The solution found was to introduce a certain number of corrugations of the outer shell of the resonator. The size, spacing and extent of these corrugations was carefully optimized to eliminate multipacting. This novel method is described in a report. [ref Naik]

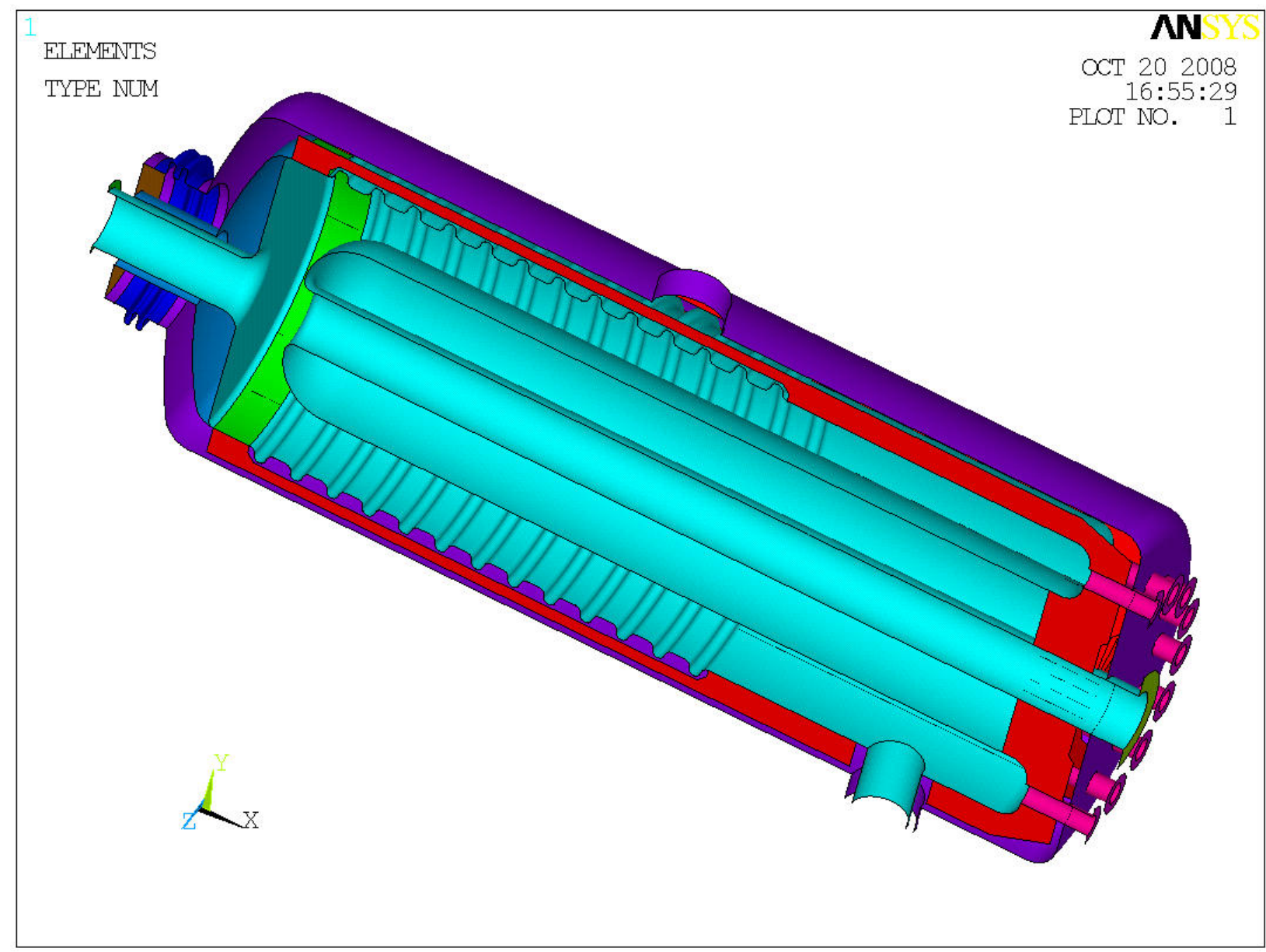

Figure 2. The 3-D shape of the $56 \mathrm{MHz}$ cavity showing the helium vessel, tuning arrangement (left side), anti-multipacting corrugations, helium vessel connections, fundamental damper port (pointing down) and the multiple ports for chemistry and various RF connections (HOM dampers, fundamental power coupler and pick-up probe) at the right side of the figure. 


\section{Mechanical and Cryogenic Properties and Tuning}

The mechanical design of the cavity has to satisfy various criteria:

a) The cavity has to be strong enough to satisfy the AMSE Pressure Vessel Code.

b) The cavity has to be rigid enough to minimize the frequency fluctuation due to helium pressure noise and environmental acoustic and seismic noise.

c) The cavity has to provide for tuning over a sufficiently large frequency range without compromising (a) and (b) above.

d) The design must allow for correcting manufacturing tolerances and bring the frequency of the cavity well within range of the tuner.

e) The design has to incorporate ports for the chemistry of the niobium and for various electromagnetic probes.

f) The design has to accommodate the helium vessel.

g) The support system should be designed to accommodate cooling to liquid helium temperature with a stable and precisely known location and orientation of the cavity.

h) The design should allow a fine tuning during the final assembly weld to bring the frequency of the cavity to the desired frequency, considering all the changes that take place during cooling, evacuation, pressure change, chemistry and welding. A frequency table [Ref Chang] provides estimates of the frequency changes related to these steps.

The mechanical design made takes all of these points into account [Ref Pai], and also includes a design of a mechanical tuner, including a slow (stepping motor) and fast (piezoelectric) drives.

It should be stated from the start that the $56 \mathrm{MHz}$ storage cavity is not designed to track the RHIC bunch frequency as it is accelerated from injection to storage energy. The tuning range is provided just for the purpose of (1) correcting for manufacturing errors, (2) controlling the voltage of the cavity by changing its frequency in relation to the bunch frequency and (3) detuning the cavity to reduce the power in the fundamental damper during it extraction.

\section{Beam Stability Considerations}

To avoid the Robinson Instability, since the cavity is operated above the RHIC transition energy, the cavity's resonance frequency should be below the bunch RF frequency.

The coupled bunch instability of the ring-cavity system has been studied [Ref Blaskiewicz, Ref Towne]. The stability study specified the necessary damping of the cavity during acceleration, in particular in passing through the transition energy, and at storage. 
As the RHIC beam is accelerated, the bunch revolution frequency changes due to the small, but nonnegligible change in the beam velocity. At injection, cavity fundamental will be heavily damped. The damper will be removed when the beam reaches store energy.

Longitudinal Coupled Bunch stability: Good damping of HOM modes provides stability at store of the longitudinal emittance of the RHIC beam.

\section{Location of the $56 \mathrm{MHz}$ Cavity in RHIC}

The cavity will be placed in the common section near IP4. That will allow one cavity to serve both blue and yellow rings. This location has the advantage that a single superconducting cavity can provide large storage bucket for both RHIC rings, saving money in construction and in operations. On the other hand, the cavity must handle double the current, thus requiring higher power handling capabilities in the fundamental mode damper.

To calculate the exact location of the cavity in the ring, we consider the following. First, the single ring bunch spacing for 120 bunch (counting the abort gap) is $31.95 \mathrm{~m}(9.383 \mathrm{MHz}$ ). The wavelength at 56.34 $\mathrm{MHz}$ is $\lambda=5.325 \mathrm{~m}$. Since the beams in the two rings travel in opposite direction, for the same storage phase, the bunches of the two rings should cross the cavity's gap with $180^{\circ}$ phase difference.

Another consideration is the density of the bunch frequency harmonic lines in the cavity. This can be reduced by increasing the effective bunch frequency with the use of two rings. Thus we aim at nearly even bunch spacing (counting both rings), to be exact $3 \lambda \pm \lambda / 2$.

Finally, we take into account the fact that when the cavity is moved from the IP a given distance, the phase difference of the two rings with respect to the cavity amounts to double the distance. Therefore, we move the cavity half of the distance for (nearly) even bunch spacing, or $1.5 \lambda-\lambda / 4$

In conclusion, the location of the cavity's gap center should be offset $6.65625 \mathrm{~m}$ from the exact IP location. 


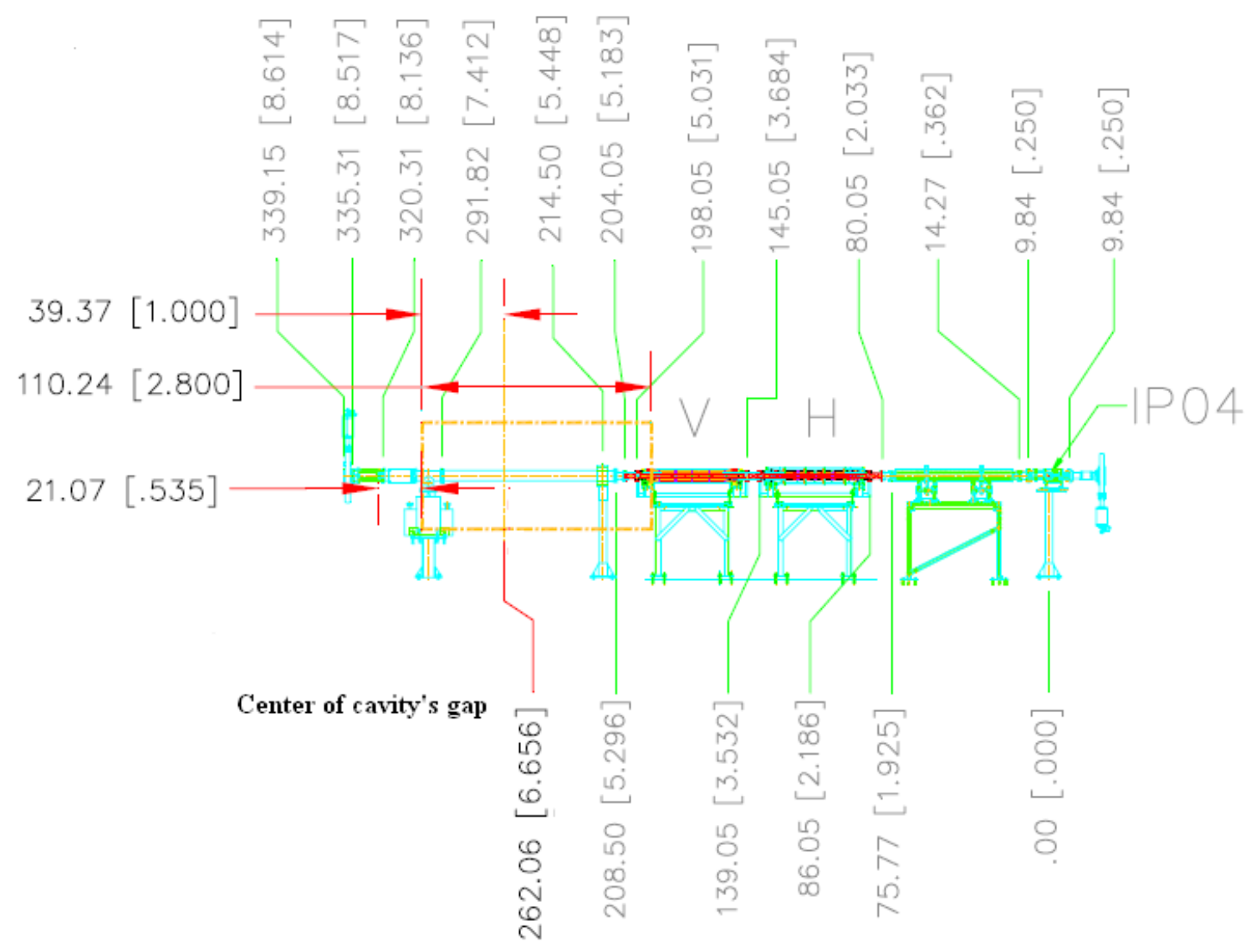

Figure 3. The location of the $56 \mathrm{MHz}$ cavity is shown in the common beam line of both the Blue and Yellow RHIC rings in Sector 3, next to IP4. The interference is with devices that will be removed by the time the $56 \mathrm{MHz}$ cavity comes for installation.

\section{The Electrical Circuit}

The fundamental mode of the resonator can be described well by an RLC equivalent circuit, allowing for a simple analysis.

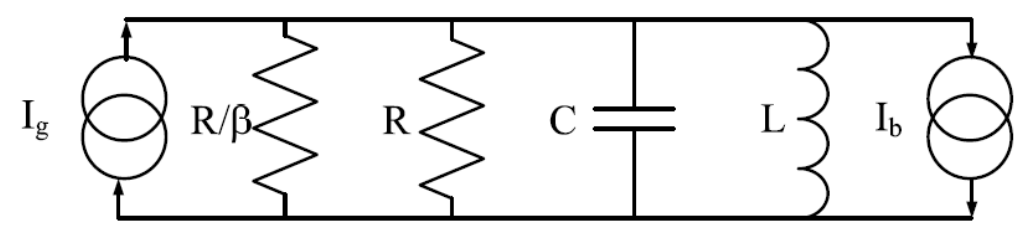

Figure 4. The electrical equivalent circuit used in the report. 


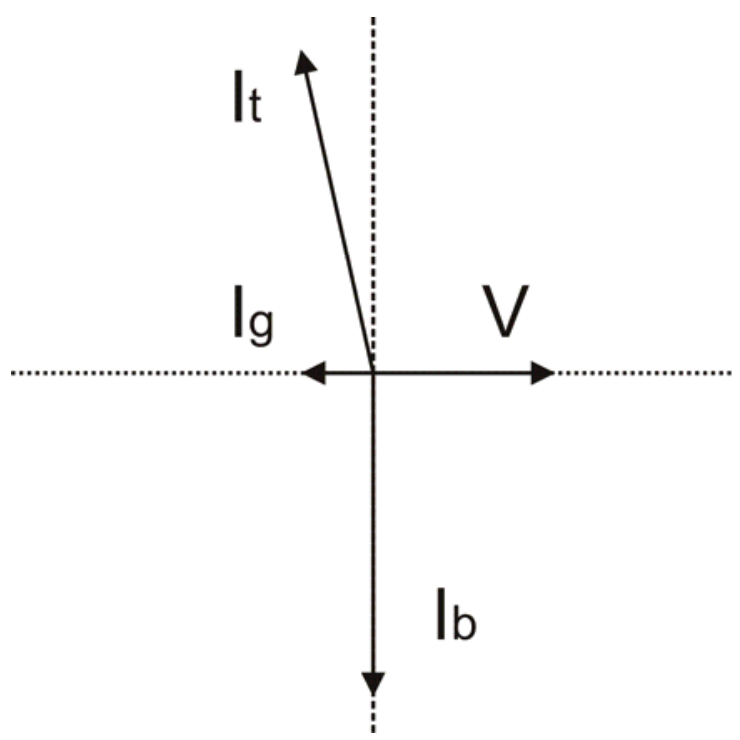

\section{Figure 5. The phasor diagram for the currents and voltage of the circuit.}

We shall be using the equivalent circuit definition shown in Figure 4 and the current and voltage phasors as shown in Figure 5. We define the real axis to be along the voltage of the capacitor. Since the resonator will be mostly beam driven, the generator current, $I_{g}$, is very small and the beam current, $l_{b}$, is very close to being 90 degrees off the voltage. In the equations below, we will show phasors with a vector notation, and a quantity written without the vector arrow is the length of the phasor.

The total current $I_{t}$ is given by

$\vec{I}_{t}=\vec{I}_{g}-\vec{I}_{b}$

where $\mathrm{I}_{\mathrm{g}}$ is the generator current and $\mathrm{I}_{\mathrm{b}}$ is the beam current. The admittance $\mathrm{Y}$ of the circuit (as measured across the capacitor's terminals) is

$\vec{Y}=\frac{\vec{I}_{t}}{V}=\frac{1+\beta}{R}+j\left(\omega C-\frac{1}{\omega L}\right)$

where $\beta$ is the coupling factor, and the generator current is related to the forward power $P$ by

$$
I_{g}=\sqrt{\frac{8 \beta P}{R}}
$$

For stability above the transition energy, the resonator is capacitive for the beam

If the resonator is not driven, than the beam will provide for the power loss in the superconductor. If there is no other cavity in the ring to provide this power, then the bucket energy will drift. Consider that there is no other cavity. Then to avoid energy drift the generator should provide just enough power to make the bucket stationary. It follows that

$$
I_{g}=\frac{1+\beta}{R} V
$$




$$
P=\frac{V^{2}(\beta+1)^{2}}{8 R \beta} \quad \text { and } \quad I_{g}=\frac{1+\beta}{R} V
$$

Of course, the minimum generator power is at $\beta=1$.

We can relate the equivalent circuit variables to the accelerator definitions of stored energy and R/Q:

$$
\frac{R}{Q}=\frac{V^{2}}{2 \omega U} \quad L=\frac{R / Q}{\omega} \quad C=\frac{1}{\omega R / Q}
$$

Let us define the detuning of the resonator off the beam frequency by $\delta$ :

$$
\delta=\frac{2 \Delta \omega}{\omega}
$$

The we obtain (where $\phi_{\mathrm{s}}$ is the beam phase relative to the voltage on the capacitor)

$$
\frac{V \delta}{R / Q}=-I_{B} \sin \left(\phi_{s}\right)
$$

Since we want a stationary bucket,

$$
\phi_{s}=-\frac{\pi}{2}
$$

then we get an important relationship for the degree of detuning we need for a given beam current and gap voltage.

$\delta=\frac{R}{Q} \frac{I_{B}}{V}$

For example, $\mathrm{I}_{\mathrm{B}}=0.5 \mathrm{~A}, \mathrm{~V}=2.5 \mathrm{MV}, \mathrm{R} / \mathrm{Q}=40$ Then $\delta=8 \times 10^{-6}, \Delta \mathrm{f} / \mathrm{f}=4 \times 10^{-6}, \Delta \mathrm{f}=224 \mathrm{~Hz}$. (Note: the maximum current with protons and both rings can reach twice this current, or about 1.1A).

Please note a few important points: The beam current in the resonator's circuit is twice the actual beam current since each particle induces twice its charge in traversing the gap. The beam current for a resonator seeing the beams of both rings has to be derived from the sum of the two beam currents. Finally, the Fourier component of the beam must be udes, since the bunch length is typically not negligible compared to the resonator's resonant frequency.

Another consideration is the effect of the abort gap. An abort gap of length $T_{\mathrm{g}}$ introduces a transient phase modulation on the stored bunches (ref Boussard, PAC91) with a maximum value of $\Delta$ 
$\delta f=\frac{1}{2} \frac{R}{Q} f \frac{I_{b}}{V} \cos \left(\phi_{b}\right)$

$\Delta=2 \pi \delta f T_{g}$

This modulation is independent of the $Q$ of the cavity, and is smaller for a larger gap voltage and smaller R/Q (both favor SRF cavities!)

\section{Frequency Correction Using In-Quadrature Power}

Starting with the total current, given as the generator current minus the beam induced current in the resonator, we have

$\vec{I}_{t}=\vec{I}_{g}-\vec{I}_{b}=V\left(\frac{\beta+1}{R}+j \frac{\delta}{R / Q}\right)$

Let us assume that the normalized frequency deviation consists of a constant part, $\delta_{0}$, and a time dependant deviation $\xi$. If we now write down the real and imaginary parts of this equation, and take the real axis to be along $V$, we get

$I_{b}=\frac{V \delta_{0}}{R / Q} \quad I_{g}^{i}=\frac{V \xi}{R / Q} \quad$ where $I_{g}^{i}$ is the imaginary part (in-quadrature component) of the generator's current. Also note that tuning out the deviation $\xi$, requires an in-quadrature component of the generator current such that:

$$
\frac{\xi}{\delta_{0}}=\frac{I_{g}^{i}}{I_{b}}
$$

Now we can optimize the fundamental power coupling coefficient $\beta$ such that we get the maximum inquadrature generator current $I_{g}^{i}$ for a given generator forward power $\mathrm{P}_{\mathrm{f}}$. We express $I_{g}^{i}$ in terms of $\beta$, equate the derivative to zero and solve for $\beta$. We get

$\beta_{o}=\frac{2 P_{f}}{P_{0}}-1$, where $P_{0}$ is the power of the unloaded resonator. Using this value of $\beta_{0}$ and solving now for $I_{g}^{i}$, we get 


$$
I_{g}^{i}=\frac{\sqrt{8 P_{f} P_{0}\left(2 P_{f} / P_{0}-2\right)}}{V}
$$

For $\beta \gg 1$ this simplifies to

$$
I_{g}^{i}=\frac{4 P_{f}}{V}
$$

Therefore the normalized deviation that can be corrected by the generator at this optimal coupling is given by

$$
\frac{\xi}{\delta_{0}}=\frac{4 P_{f}}{V I_{b}}
$$

But, substituting the value of $\delta_{0}$ from $\delta_{0}=\frac{I_{b} R / Q}{V}$, we get

$$
\xi=\frac{I_{g}^{i} R / Q}{V}, \text { thus the tuning is independent of beam current. }
$$

Replacing the generator current by the forward power provides the final tuning equation

$$
\Delta f=2 \frac{P_{f} f R / Q}{V^{2}}
$$

A numerical example, for $2.5 \mathrm{MV}$ gap voltage a $1 \mathrm{~kW}$ amplifier will provide about $0.7 \mathrm{~Hz}$ tuning range, at any beam current. This corresponds to a coupling factor of 49 , or a loaded $Q$ of about $4 \times 10^{7}$.

\section{Effect of mechanical vibrations}

Mechanically induced frequency shifts of the resonator (due to helium pressure fluctuations or environmental acoustic noise) produce small changes of amplitude and phase of the resonator. The phase change is negligible since the resonator is beam driven and the phase is near 90 degrees. However the amplitude of the voltage on the gap may change. To evaluate this effect, we neglect the amplifier power, assume that the mechanical noise modulates the capacitance and solve for the voltage under a time-dependant capacitance.

The current in the circuit is given by

$$
\vec{I}=I_{b} e^{j \omega t}=\frac{d}{d t}(\vec{V} C)+\frac{\vec{V}}{R}+\frac{1}{L} \int \vec{V} d t
$$


where the capacitance is time dependant

$$
C=C_{0}\left(1+\mu\left(e^{j \omega_{m} t}+e^{-j \omega_{m} t}\right)\right)
$$

where $\mu$ is half the amplitude of the sinusoidal modulation of the capacitor, which has a frequency $\omega_{\mathrm{m}}$.

$$
\frac{d}{d t} C=j C_{0} \mu \omega_{m}\left(e^{j \omega_{m} t}-e^{-j \omega_{m} t}\right)
$$

We assume that the voltage is composed of the carrier and two sidebands, at angular frequencies

$$
\omega_{ \pm}=\omega \pm \omega_{m}
$$

$\vec{V}=V_{0} e^{j \omega t}+V_{-} e^{j \omega_{-} t}+V_{+} e^{j \omega_{+} t}$

Therefore the time derivative and integral of the voltage are given by

$$
\begin{aligned}
& \frac{d}{d t} \vec{V}=j \omega V_{0} e^{j \omega t}+j \omega_{-} V_{-} e^{j \omega_{-} t}+j \omega_{+} V_{+} e^{j \omega_{+} t} \\
& \int \vec{V} d t=\frac{V_{0}}{j \omega} e^{j \omega t}+\frac{V_{-}}{j \omega_{-}} e^{j \omega_{-} t}+\frac{V_{+}}{j \omega_{+}} e^{j \omega_{+} t}
\end{aligned}
$$

We can substitute the values given above for the voltage, capacitance and their derivatives or integrals. Then we collect the terms for a particular sideband, say the upper sideband, and this results in the following connection between the amplitude of the carrier and that of the sidebands:

$$
\frac{V_{ \pm}}{V_{0}}=-\frac{j R C_{0} \mu \omega}{1+2 j Q \frac{\Delta \omega \pm \omega_{m}}{\omega}}
$$

where $\Delta \omega=\omega-\omega_{\mathrm{r}}$ is the detuning of the resonator from the beam frequency, and this is a positive quantity since the resonator is below the beam frequency. With a bit more manipulation we get

$$
\left|\frac{V_{ \pm}}{V_{0}}\right|=\frac{\mu Q}{\sqrt{1+4 Q^{2}\left(\frac{\Delta \omega \pm \omega_{m}}{\omega}\right)^{2}}}
$$

Thus, to avoid a large disturbance of the voltage by the sidebands, $\mu$ should be small compared to $1 / Q$, particularly when a sideband nulls the detuning. 
Assuming a frequency deviation of $\pm 0.56 \mathrm{~Hz}$, then $\mu^{\sim} 10^{-8}$. For a loaded $Q$ of $4 \times 10^{7}$, the worst value of the sideband amplitude ratio is 0.4 , and this is only for a small band of mechanical frequencies for which the mechanical frequency $\omega_{m}$ cancels $\Delta \omega$ within about one Hertz. The requirement for the amplitude stabilization is that it be better than $10^{-4}$ at frequencies of about double the synchrotron frequency of RHIC (about $240 \mathrm{~Hz}$ with the $56 \mathrm{MHz}$ cavity at $2.5 \mathrm{MV}$ ). At this frequency the noise content is expected to be much less than $0.5 \mathrm{~Hz}$. 